\title{
Time to measure up
}

\section{As electronic devices continue to evolve, the metrology methods required to characterize them also need to evolve.}

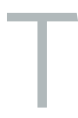
he International System of Units (SI) has seven base units: the metre, the second, the mole, the ampere, the kelvin, the candela and the kilogram. As of 20 May, four of these - the kilogram, the kelvin, the ampere and the mole have been redefined. For the kilogram, which since 1889 has been defined using a cylinder of platinum-iridium alloy held in a laboratory in the suburbs of Paris, the change means that it is now defined in terms of the Planck constant. The revisions also mean that all seven base units are now defined according to physical constants, rather than physical objects or arbitrary references.

The launch of these new definitions was timed to coincide with World Metrology Day, an annual celebration of the signing of the Metre Convention on 20 May 1875. Metrology is the science of measurement (and not to be confused with the similar sounding meteorology, the science of the atmosphere). For electronics, metrology becomes increasingly critical as devices shrink in size and become more complex in shape. As George Orji and colleagues highlighted in a recent review of metrology methods for integrated circuits ${ }^{1}$, the gate length of devices is projected to reach $6 \mathrm{~nm}$ by 2024 , and by then gates may wrap around vertical nanowires. Optimized techniques to manufacture such devices are being developed. But what exactly the methods are that can characterize these devices remains uncertain.

Metrology plays a role in all of the different stages of integrated circuit manufacturing, from exploratory research to technology development to process control ${ }^{2}$. It thus requires instruments that use a variety of different fundamental probes, including light, electrons and $\mathrm{X}$-rays, and measure a variety of different parameters, including critical dimensions, surface properties and defects. For dimensional metrology, techniques such as scanning electron microscopy (SEM), small-angle X-ray scattering (SAXS), and atomic force microscopy (AFM) can be used. As Orji and colleagues explore in their review $^{1}$, no one-dimensional metrology technique can provide all of the capabilities required to adequately characterize complex devices. Therefore, hybrid metrology, in which methods (such as SAXS and SEM, or SEM and AFM) are combined, is a key area of development. The researchers also highlight two emerging technologies that

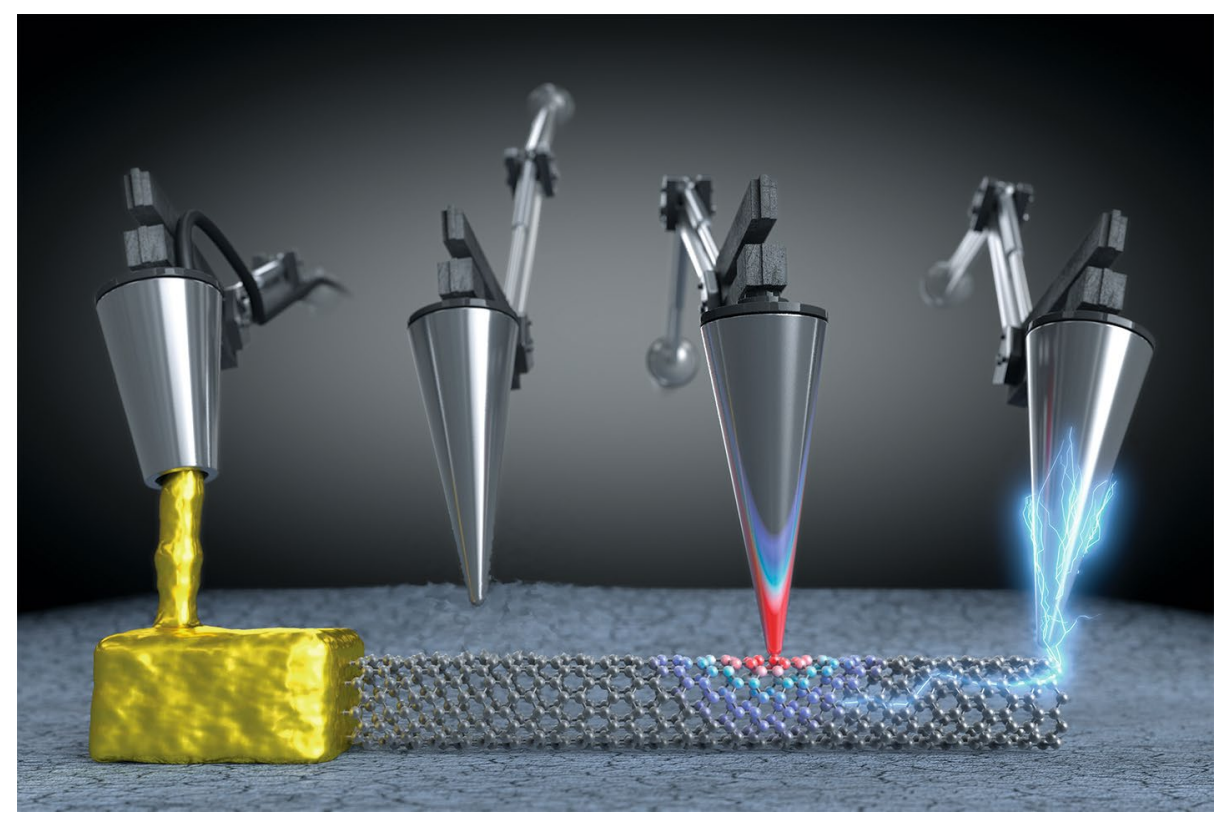

A schematic illustration of a potential multi-probe scanning probe microscopy technique that could be used to carry out multiple experiments simultaneously. Credit: Springer Nature Ltd.

could have a profound effect on the future of integrated circuit metrology: advanced data analytics, which uses techniques such as deep learning to examine large data sets; and sub-wavelength imaging methods, such as plasmonic-assisted optical focusing or superresolution microscopy, which can image beyond classical diffraction limits.

When it comes to assessing the performance of an electronic device, a probe station connected to a semiconductor parameter analyser is often used, a setup that can apply electrical stresses to the device and extract the required figures of merit. However, as device sizes shrink, techniques capable of increasingly precise and sophisticated measurements are called for. In a Perspective in this issue of Nature Electronics, Fei Hui and Mario Lanza argue that conductive atomic force microscopy (CAFM) has the potential to play a critical role here.

Electrical measurements using AFM were first reported in 1993: a modified microscope that had a cantilever with a silicon tip coated with titanium was used to examine the dielectric strength of silicon dioxide films ${ }^{3}$. Since then, CAFM has provided various insights into nanoscale materials and devices. But, as Hui and Lanza explain, its capabilities remain relatively limited compared to probe stations, and the full potential of the technique has not yet been realized. They highlight a number of areas in which the technique could be improved: novel characterization methods, such as four-dimensional imaging (current maps at different depths of a material) or electromechanical tests for flexible electronics; upgraded electronics that can apply advanced electrical stresses such as ramped voltage stresses with high current; and enhanced multi-probe approaches.

The researchers go on to envisage an intricate multi-probe setup that could simultaneously fabricate and characterize nanoscale devices. Much remains to be done before such a system could become a reality. But as electronic devices continue to evolve, metrology methods - be it the scanning probe systems outlined by Hui and Lanza, or the hybrid and emerging technologies detailed by Orji and colleagues - need to evolve with them.

Published online: 17 June 2019

https://doi.org/10.1038/s41928-019-0269-3

References

1. Orii, N. G. et al. Nat. Electron. 1, 532-547 (2018).

2. Ma, Z. \& Seiler, D. G. (eds) Metrology and Diagnostic Techniques for Nanoelectronics (Pan Sanford, 2017).

3. Murrell, M. P. et al. Appl. Phys. Lett. 62, 786-788 (1993). 